\title{
Comparison Between the Methods of Adjustment and Constant Stimuli for the Estimation of Redirection Detection Thresholds
}

\author{
Weiya $\mathrm{Chen}^{1 \star}$, Nicolas Ladevèze ${ }^{2}$, Wei $\mathrm{Hu}^{1}$, Shiqi Ou ${ }^{1 \star}$, and Patrick Bourdot ${ }^{2 \star}$ \\ 1 School of Software Engineering, Huazhong University of Science and Technology, \\ Wuhan, China \\ weiya_chen@hust.edu.cn, oushiqi@isyslab.org \\ 2 VENISE Team, LIMSI, CNRS, Univ. Paris-Sud, Université Paris-Saclay, Orsay, \\ France \\ patrick.bourdot@limsi.fr
}

\begin{abstract}
Redirection Detection Thresholds (RDTs) are defined to represent the limits of undetectable gains and serve as important input parameters for redirected walking algorithms. However, it is not trivial to get a user's RDT estimation in a few trials with existing methods such as the commonly used Method of Constant Stimuli (MCS). In aim to achieve efficient RDT estimation, we chose a classic psychophysical method - the Method of Adjustment (MoA), and compared it against MCS with a series of within-subject experiments respectively on translation, rotation and curvature gains. The results show that MoA gets overall similar RDT estimations with MCS over the same population, except for some systematic differences on translation and rotation gains. Moreover, MoA (with 20 trials) saves about $33 \%$ experiment time when compared against MCS, and has the potential to save more as the results of MoA remain relatively stable when the number of trials decreases. Further studies are needed to compare MoA with adaptive methods and to discover the potential relationship between RDTs and perception traits at individual level.
\end{abstract}

Keywords: Navigation · Redirected Walking · Detection Threshold · Method of Adjustment.

\section{Introduction}

Exploring large virtual worlds by natural walking within limited physical workspace is still an open research problem in Virtual Reality and related fields. Unlike walk-in-place [31] or other motion-based steering metaphors [2], natural walking is considered to yield best subjective presence [32].

\footnotetext{
* This is the authors' version of the work. It is posted here for your personal use. Not for redistribution. The definitive version of record was published in Lecture Notes in Computer Science (Springer), https://doi.org/10.1007/978-3-030-31908-3 4
} 
Besides sophisticated walking hardware, many software solutions were developed to enable natural infinite walking with lower cost and easier hardware deployment [30]. Most existing techniques either have limited applicable virtual scenes, or somehow interrupt users' ongoing task which may lead to the break of presence. In searching for redirection methods that minimize artificial interventions during walking, Razzaque et al. [24] developed another method called redirected walking. It allows users to walk through a large virtual world while keeping their real-world path inside a much smaller region than the virtual counterpart. It leverages users' tolerance of subtle visual-vestibular conflicts to decouple their virtual and physical paths while a user walks in the virtual environment. In general, three types of gains - translation, rotation and curvature gains [29] - can be allocated at specific moments by algorithms using reactive [11] or predictive control strategies [16].

Redirected walking has been actively developed and is pushed forward by advances from both virtual reality and science of perception for more than ten years [20]. Besides designing algorithms for optimal gain distribution during walking, the main question to be answered for redirected walking is - how much can we redirect the users before they notice the gains? To answer this question, the Redirection Detection Thresholds (RDTs) are defined to represent the limits of undetectable gains and serve as important input parameters for redirected walking algorithms. Any type of redirection gain applied at a given moment shall not go over the corresponding RDT in order to remain imperceptible (although being perceptible will not necessarily lead to the break of presence as shown recently [27]).

To understand the mechanism of gain detection, existing studies begun by measuring RDTs with psychophysical experiments [23]. Since the comprehensive study by Steinicke et al. [28], many follow-up studies repeated the measurement of RDTs with the same estimation method, but under different experimental conditions [14]. The mainstream estimation method used in these experiments is the Method of Constant Stimuli combined with a Two-Alternative Forced Choice task (MCS-2AFC). The MCS can help us to draw the whole psychometric curve for a given subject, but at the cost of large number of trials.

The use of MCS as a threshold estimation method leads to several problems due to its high time cost: first, it is difficult to scale up the experiments by involving more subjects or testing different factors that may have potential influence on RDTs. Second, it is difficult, even impossible to collect data from people having trouble with long-exposure VR experience (e.g. cybersickness). Moreover, as the RDTs appear to be user-dependent [1], a time-saving RDT evaluation method can help to provide users with personalized RDTs through calibration instead of the average thresholds from all users. As a consequence, we need a more time-efficient evaluation method that allows us to make quick estimation of RDTs.

In this paper, we present a series of experiments using a classical psychophysical method - the Method of Adjustment (MoA) to make quick assessment of RDTs for redirected walking. The reason for choosing MoA is that it is intu- 
itively appealing, easy to set up, and in general requires much fewer test trials than other classical or adaptive psychophysical methods.

We conducted a group of experiments using MoA and another group with MCS-2AFC on the same group of subjects. Through the comparison, the goal is not to find the "better" method for RDT estimation, but rather to see whether a time-efficient method like MoA yields similar results with MCS over the same population, despite their inherent methodological differences. The experimental design and preliminary results were briefly reported in [5]. Here we detail all the experimental settings and conditions, along with results and in-depth discussions, that shall serve as a basis for future studies. Moreover, with the aim of understanding the between-user variability of RDTs, we report a series of tests we conducted to collect users' perception traits related to space and motion. We conclude that further correlation analyses between such perception traits and RDT identification tests are necessary.

\section{Related Work}

The study of RDTs actually concerns the measurement of subjective sensation in response to physical stimuli, so we can use psychophysical theories and tools to get estimations of RDTs.

In fact, the estimation of RDTs is more complex than other perceptionrelated tasks (e.g. size or color discrimination) as we are comparing the stimuli from visual and non-visual channels - at least three different sensory channels (visual, vestibular and proprioceptive) of human sensorimotor system are involved in the walking process.

Here we first briefly present the three classical psychophysical methods, then describe in detail how they have been applied for the estimation of RDTs, and finally some existing observations on the between-subject variation of RDTs.

\subsection{Threshold Estimation in Psychophysics}

Threshold estimation is one of the fundamental tasks in Psychophysics. Two types of thresholds are defined: Absolute Threshold (or Absolute Limen) - the intensity at which a stimulus becomes detectable, and the Difference Threshold (or Difference Limen) - the difference in intensities at which an observer can notice the differences.

No matter the type of threshold that we are targeting, threshold estimation experiments are composed of tasks and sampling methods [7]:

- The task: it can be either a detection ("yes/no" questions) or discrimination (e.g. forced choice) task. In general forced choice is considered more robust as compared to "yes/no" questions by eliminating response bias.

- The sampling methods: they define the way that stimuli intensities are presented to the subject. The three classical sampling methods are the Method of Constant Stimuli (MCS), the Method of Limits (MoL) and the Method of Adjustment (MoA). 
MCS is characterized by large number of trials with equally spaced levels of stimulus intensities in a randomized order, whereas MoL uses alternate ascending and descending trials and the threshold is taken as the average of the transition points from each series. MoA is quite similar with MoL in term of intensity sampling, except that the subjects are given direct control of the stimulus intensity [8]. Being the most intuitive and efficient method among the three, MoA is often used to get initial estimates of thresholds.

It is known that threshold values depend in part on the employed experimental method [10]. Many studies [22,34] reported the difference of threshold values obtained with these methods for different research purposes, but were careful about their recommendations on choosing the "best" method because there was no common rule found for the thresholds and the results seemed to be experiment-dependent.

\subsection{RDT Estimation Overview}

With the inherent complexity of walking, it is unlikely that one could get a clear picture of the whole process from a single experiment, our knowledge about RDTs had grown incrementally as more experiments were conducted.

Razzaque [23] conducted several initial experiments on RDT estimation, mostly on scene rotation detection with adaptive 2-track staircase methods (20 trials). These experiments were conducted with limited number of subjects and trials, and the results turned out to have wide confidence interval.

Steinicke et al. [28] conducted the first formal study on RDT evaluation by a series of psychophysical experiments in which RDTs for translation, rotation and curvature gains were tested separately. The evaluation method used was a combination of MCS and 2AFC. They describe the experiments as 2AFC experiments for simplicity (as commented by Grechkin et al. [9], the 2AFC used here is actually a kind of pseudo-2AFC task since subjects didn't choose between a test signal and a reference signal as in traditional $2 \mathrm{AFC}$ tasks), and found that the detection thresholds for translation gain were $[0.86,1.26]$, similarly the detection thresholds for rotation gains were $[0.67,1.24]$ and a curvature gain of no more than 0.045 was considered unnoticeable.

Many following experiments employed the MCS-2AFC method for RDT estimation, but instead of studying all three kinds of gains, they often focused on one type of redirection gain and found that RDTs could vary under different testing conditions [33,14]. For example, the threshold for translation gain was smaller with the presence of virtual feet [12], the threshold for rotation gains was different when users were sitting in a wheelchair [3], or exposed to different level of visual densities [21]. For the curvature gains, the corresponding threshold could interact with factors such as ambient noise [23], walking speed [17] and cognitive load [4], etc.

It would be interesting to scale up the experiments mentioned above for all types of redirection gains, or to check the interaction between these factors. However, the MCS-2AFC method is too time consuming for such purpose. So 
researchers started to work with more light-weight adaptive methods. For example, Grechkin et al. [9] studied the interaction between translation and curvature gains by using Green's maximum likelihood procedure, which is a variant of the adaptive method combined with a "yes/no" task. Nguyen et al. [19] used a two interleaved 2AFC task combined with a three-down/one-up staircase method with fixed step size for left and right directions.

The adaptive methods are surely more efficient than MCS as they require less trials, but it is more complex to make design choices with numerous variants and parameters as compared to MCS and MoA. Another potential bias using adaptive methods is that participants are less likely to detect large gains when the gain value increases progressively from trial to trial, because it is known that users adapt to increasing redirection gains to become less sensitive to the manipulation over time [9]. Considering the two drawbacks that we just highlighted for adaptive methods, we choose to study the usability of MoA for the estimation of RDTs.

\subsection{The Between-subject Variability of RDTs}

An observation can be made from existing studies that the reported RDTs to some extent all differ from one to another, which leads researchers to look into the exact cause of such diversity. Besides methodological differences, Nguyen et al. [19] proposed to put different factors into two groups: intrinsic and extrinsic. Intrinsic factors are specific to a person's perception trait (e.g. visual dependence, sensitivity to visual-vestibular conflict, etc.), while extrinsic factors include user-related factors (engagement, cognitive load, etc.), environment design (e.g. presence of virtual feet, visual density, etc.) and hardware setup (HMD intrinsics, tracking fidelity, etc.) [1].

Recent research work has just begun to study the between-subject variability of RDTs. For example, Nguyen et al.'s experiment [19] shows that on average women have higher detection thresholds for curvature gains than men. Rothacher et al. [26] find that the threshold for curvature gains is positively correlated with a user's visual dependency, though they did not test other types of redirection gains in this experiment.

The study of the relationship between intrinsic factors and RDTs is not only useful for building a user-specific profile in order to provide better user experience, but can also help us to understand the underlying model of sensory fusion during redirected walking.

\section{$3 \quad$ Experiments}

Since MoA is an efficient classical sampling method in psychophysics, but has not yet been formally used in the context of RDT estimation, we designed the following experiments to see how its results differ from those of MCS-2AFC (which is believed to be the most reliable method, and hereafter referred as MCS for simplicity) on the same population. In addition, we examined the correlation 
between each user's perception trait and their RDTs in order to see how RDTs differ between individuals. Experimental protocol and results for all experiments are presented in this section.

\subsection{Participants}

In total 24 graduate students participated our experiments (17 male and 7 female) with an average age of 23 years old $(+/-0.8)$. All participants have normal or corrected to normal vision. Besides age and sex, we collected their familiarity with video game and virtual reality devices using questions with a 4-point rating scale, ranging from 1 (none), 2 (a little), 3 (sometimes), 4 (often). The average score of video game experience is relatively high $($ mean $=3.04, \mathrm{sd}=1.04)$ since only two of them have never played video games. On the contrary, most participants have little experience with virtual reality devices (mean $=1.67, \mathrm{sd}=0.87$ ).

All participants had no experience with redirected walking and were naive about the purpose of the experiments. We used a within-subject design for the comparison between results from experiments with MCS and MoA. These two groups of experiments were counterbalanced to reduce order effects and were arranged on different days. Each group of experiments contained three sub-experiments (translation, rotation and curvature) that were also counterbalanced. Subjects participated a training session of about 10 minutes to get familiar with the hardware setup and experiment procedures, they were allowed to take breaks at any time during the experiments. Although no official research ethics committee is yet available in our establishment, all participants were recruited and treated in accordance with the principles outlined in the Declaration of Helsinki.

\subsection{Experimental Setup}

The experiments were carried out in a lab room with a standard commercial VR setup (HTC Vive Pro) that contains an HMD $(1400 \times 1600$ pixels per eye, $90 \mathrm{~Hz}$ refresh rate, 110 field of view) and two handheld controllers. During the experiments, subjects wore the HMD and held one controller as input device (see Figure 1).

The two base stations used for tracking were placed at a distance of $5.5 \mathrm{~m}$ (slightly larger than the specified $5 \mathrm{~m}$ limit) forming a valid rectangular tracking zone of $3 m \times 4 m$ with a safety margin of $0.3 \mathrm{~m}$ to the walls. Users were able to cover the whole tracking area with a cable $(5 \mathrm{~m})$ attached to the headset from a workstation positioned on one side of the workspace.

The workstation (Intel Core i7 7700K CPU, 32GB RAM, GeForce GTX $1080 \mathrm{Ti}$ ) was fully VR compatible and was able to run the simulation at $90 \mathrm{~Hz}$. The virtual scene was a futuristic city ${ }^{3}$ rendered by Unity 3D. Only a small part of the whole city was used for our experiments (see Figure 2), its asymmetric configuration and many animation effects in the surroundings allowed users

\footnotetext{
${ }^{3}$ https://assetstore.unity.com/packages/3d/environments/sci-fi/pbr-sky-city-62261
} 


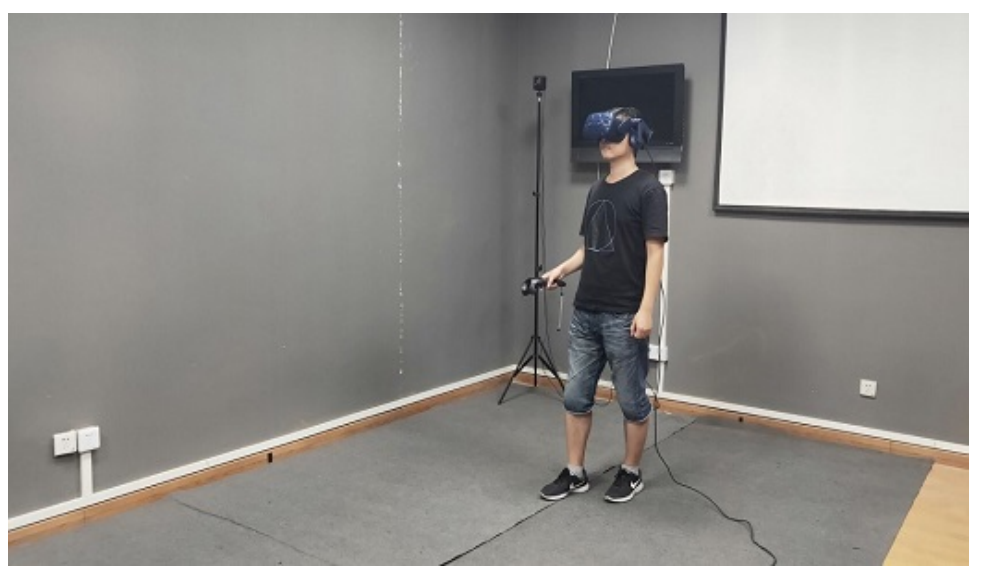

Fig. 1. A user walked in the physical workspace with an HMD and a handheld controller.

to quickly locate themselves with respect to the landmarks around. We coded the redirection control and the experiment process with $\mathrm{C} \#$ scripts on top of SteamVR components.

\subsection{Measures}

RDT estimation In existing literature, RDTs were mostly reported in form of a tuple of lower and upper detection limits. This form is intuitive as it directly shows the usable interval of undetectable gains for the redirection controller. Here we took a different representation of the threshold: a combination of the Point of Subjective Equality (PSE) and the Interval of Uncertainty (IU) [6].

The RDT for a user could be written in form of [PSE-IU/2, PSE+IU/2]. As found in previous studies, the PSE seldom meets exactly the theoretical equivalence point (i.e. gain=1) which leads to a bias (positive or negative) on gain perception, for example, a user with a PSE higher than 1 tends to be more sensitive to smaller gains $(<1)$ than larger ones $(>1)$. The IU is another important factor characterizing user's gain detection ability, for example, a user with high IU provides more room for maneuver to the redirection system than those who have lower ones.

MCS and MoA employ very different ways to compute PSE and IU. The former gets PSE and IU indirectly from the fitted psychometric curve. We used the same logistic function as described in [28]:

$$
p=f(x)=\frac{1}{1+e^{a x+b}}
$$

Here $x$ is the applied redirection gain and $p$ is the probability that the user considers the amplitude of real locomotion to be greater than the virtual counterpart. This probability is computed by counting the "real is greater" trials 


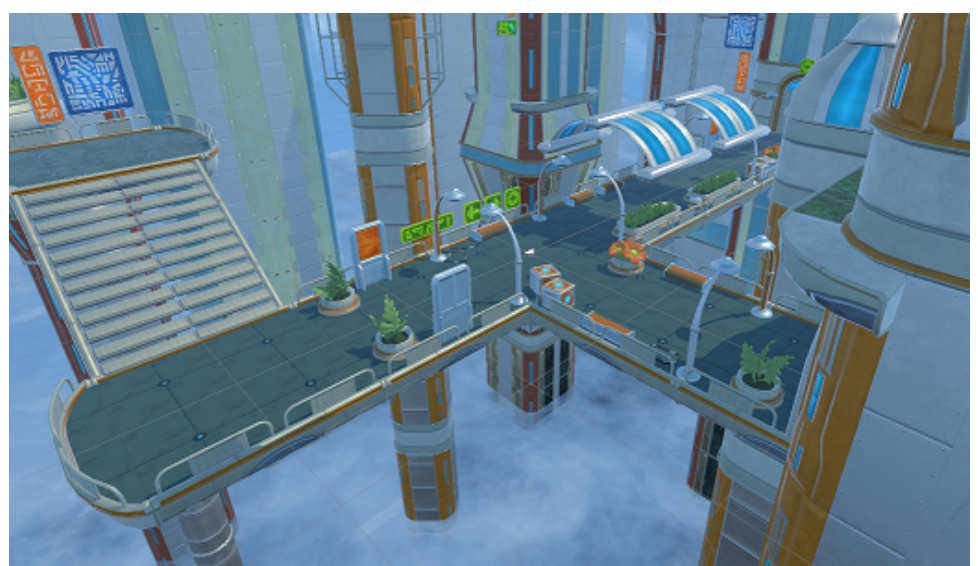

Fig. 2. Overview of the T-formed street in a futuristic city used for walking in our experiments.

divided by the total trial number per gain value. $a$ and $b$ are parameters setting by curve fitting.

The PSE corresponds to the gain value of $f^{-1}(0.5)$. IU is bounded by a lower detection threshold $f^{-1}(0.25)$ and a upper bound $f^{-1}(0.75)$, which is two times the Just Noticeable Difference (JND). Here are the equations for computing PSE (2) and IU (3):

$$
\begin{gathered}
P S E=f^{-1}(0.5)=-\frac{b}{a} \\
I U=f^{-1}(0.75)-f^{-1}(0.25)=2 \cdot J N D=-\frac{2 \cdot \ln 3}{a}
\end{gathered}
$$

Unlike MCS, MoA directly asks users to manually adjust the magnitude of redirection gain from a random starting value till no difference can be detected between visual and non-visual stimuli.

MoA contains equal number of ascending and descending trials that are tested alternatively in order to reduce estimation error. The computation of PSE (Equation 4) and IU (Equation 5) for MoA is described as follows:

$$
\begin{gathered}
P S E=\mu(g) \\
I U=2 \cdot J N D=2 \cdot z(0.75) \cdot \sigma(g)=1.349 \cdot \sigma(g)
\end{gathered}
$$

$\mathrm{g}$ is the gain value submitted at the end of each trial, $\mu$ and $\sigma$ are the mean and standard deviation of submitted gain values. JND is given by multiplying the standard deviation of PSEs by a z score of probability of 0.75 (about 0.6745) [13].

In addition to PSE and IU, we also collected each subject's mean walking speed during trials for translation and curvature gains. Since Neth et al. [17] 
found that the sensitivity to walking on a curved path was significantly lower for slower walking speeds. The walking speed data may provide cues to help us understand the obtained detection thresholds.

Perception Trait Since all subjects were tested under the same experimental protocol and condition, their RDTs should be mostly influenced by intrinsic factors such as each individual's perception trait. So we conducted a series of tests on each subject to collect their spatial and motion perception ability before starting experiments for RDTs identification.

Here we conducted tests respectively for egocentric distance and orientation perception and several other tests recommended by Ngoc et al. [18] (i.e. the Rod \& Frame, Romberg and vection test) to measure a user's visual dependence during sensory integration, which are listed in Table 1. Here are some implementation details of these tests:

Group A We assume that the error of users' egocentric distance and orientation estimation is positively correlated with RDT for translation and rotation. For distance perception, we employed a visually directed action method - blind walking [15], to assess subject's distance perception accuracy. Each subject walked 5 times with each time a random distance ranging from $2.5 \mathrm{~m}$ to $4.5 \mathrm{~m}$ (interval of $0.5 \mathrm{~m}$ ). The final score is the root mean square error (RMSE) of all trials.

The spatial orientation test was conducted in a similar way. Each subject was tested 8 times with angles ranging from 30 to 120 degrees (with interval of 30 degrees and left/right symmetry).

Group B The Rod \& Frame test experiment followed the standard procedure of the original test [35] except that the rod and frame were displayed through the HMD. We tested five different angles for the frame: $0^{\circ}, 3^{\circ}, 15^{\circ}, 27^{\circ}, 42^{\circ}$, each tested twice in a random order. The final score is also the RMS error of all trials.

In the Romberg's test [25], the subject was asked to stand with feet together on the Wii balance board ${ }^{4}$, hands by the sides, eyes open and then closed for respectively 20 seconds. The final score is the ratio between deviations of a subject's center of pressure (CoP) with eyes open and eyes closed. We also noted the deviations of CoP with eyes closed as an additional score (put in Group A) to describe a subject's profile regarding non-visual balance control.

The vection test was performed by asking the subject to stand inside a rotating optokinetic drum with constant speed (shown through an HMD). We tested three rotation speed - 20, 30 and $40 \mathrm{deg} / \mathrm{s}$ - each for twice in a random order. The time from the beginning till the subject reported self-motion was logged (a limit of $45 \mathrm{~s}$ was used if no self-motion was reported).

\footnotetext{
${ }^{4}$ https://en.wikipedia.org/wiki/Wii_Balance_Board
} 
Table 1. A summary of tests for perception trait.

\begin{tabular}{ll}
\hline group & test description \\
\hline A - non-visual perception & (1) ego-distance: blind walking \\
& (3) balance eye-closed: control of CoP \\
\hline & (4) Rod \& Frame: vertical alignment \\
B - visual dependence & (5) Romberg's ratio: control of CoP \\
& (6) vection: on-set time for self-motion \\
\hline
\end{tabular}

\section{Experiment MCS}

In this experiment, we tried to identify users' RDTs for different types of gains translation, rotation and curvature. This experiment primarily serves as a baseline for the other experiment with MoA since MCS is considered to be more robust against various bias.

\subsection{Procedure}

We followed similar procedure of Steinicke et al.'s previous experiment [28]. Figure 3 shows a subject's first person view of the virtual street used for RDT evaluation. In the translation and curvature sub-experiments, for each trial, the subject begun by standing on the starting point, then walked towards the target (a green floating sphere) till it changed color. In the rotation sub-experiment, the subject started from a fixed orientation and then rotated in place towards the target sphere till it changed color. After each trial, a question was shown in a pop-up window that allowed the subject to make the forced choice.

The detailed settings of each sub-experiment is shown in Table 2. It should be noted that the curvature gain is expressed by angles, here it is actually the amount of direction change after walking $3 \mathrm{~m}$ straight in the virtual world. The actual curvature gain can be computed by Equation 6 :

$$
g_{c}=\frac{1}{r} \approx \frac{\pi \cdot \theta}{180 \cdot d}
$$

where $\mathrm{r}$ is the radius of the corresponding circle, $\theta$ is the direction change expressed in degree and $\mathrm{d}$ is the virtual walking distance (not exactly the arc length).

For the curvature sub-experiment, we used a small step $\left(5^{\circ}\right.$ interval $)$ at gains in range $\left[0^{\circ}, 20^{\circ}\right]$ and a bigger one $\left(10^{\circ}\right)$ for gains larger than $20^{\circ}$. We also measured left and right turns separately so each angle was tested five times.

This experiment differs from the one of Steinicke et al. on two aspects: First, since the physical workspace available was quite small, in the translation subexperiment we fixed the virtual travel distance to be $2.5 \mathrm{~m}$ and the real walking distance varied according to the gain value. No "redirection-free" pre-walking was possible due to the space limitation. Second, Steinicke et al. got estimates of RDTs from the average samples of all subjects instead of fitting individual 

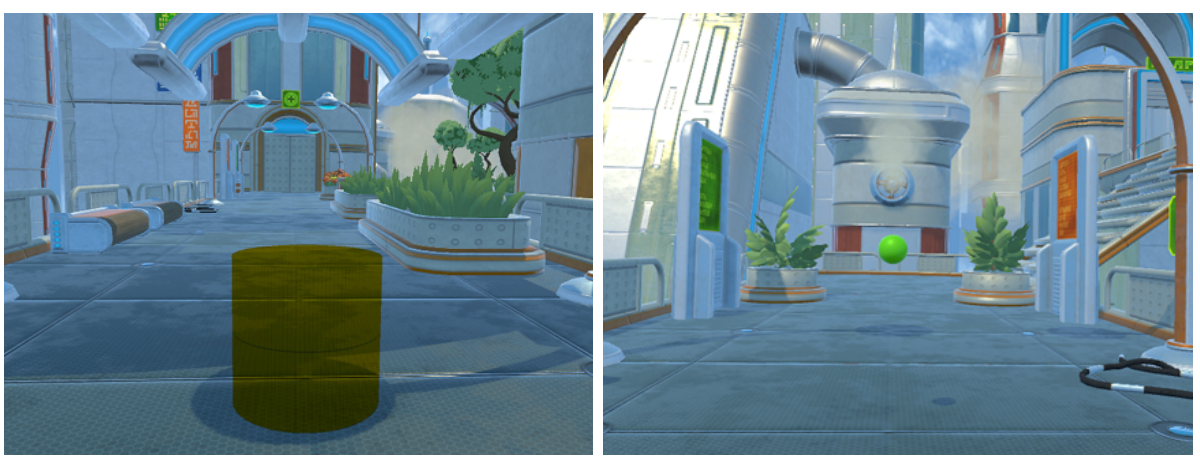

Fig. 3. The subject's first-person view in Experiment MCS. Left: the orange cylinder is the starting point for the subject to step on; Right: the green target sphere.

Table 2. Detailed settings used in Experiment MCS.

\begin{tabular}{cccc}
\hline Parameters & Translation Rotation Curvature \\
\hline Lower limit of gains & 0.6 & 0.5 & $0^{\circ}$ \\
Upper limit of gains & 1.4 & 1.5 & $50^{\circ}$ \\
Step of gains & 0.1 & 0.1 & $5-10^{\circ}$ \\
Trials per gain value & 10 & 10 & 5 \\
Total number of trials & 90 & 110 & 75 \\
Virtual walking distance/rotation angle & $2.5 \mathrm{~m}$ & $\pm 90^{\circ}$ & $3 \mathrm{~m}$ \\
\hline
\end{tabular}

psychometric functions. Here we fitted the psychometric curve for each subject so we can get a per-subject RDT estimation.

\subsection{Results}

We employed the logistic function in Equation 1 to fit the psychometric curve of each subject. The mean and standard deviation of RDTs, experiment duration and walking speed are presented in Table 3 .

All 24 subjects finished all the trials, however, two of them got data unable to be fitted by a logistic function under all three sub-experiments. The rotation and curvature sub-experiments had respectively three more subjects that were not included in the final results shown in Table 3. The data for these subjects

Table 3. The mean and standard deviation for the results of Experiment MCS.

\begin{tabular}{cccc}
\hline & Translation & Rotation & Curvature \\
\hline Number of subjects & 22 & 19 & 19 \\
PSE & $0.95 \pm 0.13$ & $1.03 \pm 0.22$ & $0.01 \pm 0.06$ \\
IU & $0.23 \pm 0.16$ & $0.5 \pm 0.34$ & $0.31 \pm 0.48$ \\
Upper \& lower bounds & {$[0.83,1.06]$} & {$[0.78,1.28]$} & {$[-0.14,0.17]$} \\
duration (minutes) & $26.8 \pm 5.3$ & $25.5 \pm 3.8$ & $24.3 \pm 4.5$ \\
walking speed (m/s) & $0.62 \pm 0.17$ & $/$ & $0.61 \pm 0.12$ \\
\hline
\end{tabular}


Table 4. Detailed settings used in Experiment MoA.

\begin{tabular}{|c|c|c|c|}
\hline Parameters & Translation & Rotation & Curvature \\
\hline Lower limit of gains & 0.6 & 0.5 & $0^{\circ}$ \\
\hline Upper limit of gains & 1.4 & 1.5 & $\pm 50^{\circ}$ \\
\hline Step of gains & 0.01 & 0.01 & $1^{\circ}$ \\
\hline Total number of trials & 20 & 20 & 20 \\
\hline
\end{tabular}

either resulted in abnormal fitted thresholds (e.g. $P S E<0$ for translation and rotation) or were even unable to be fitted by a sigmoid curve.

The detection threshold $D T_{c}($ left $)=-0.14$ and $D T_{c}($ right $)=0.17$ correspond respectively to a radius of $7.1 \mathrm{~m}$ and $5.9 \mathrm{~m}$, or $8.02^{\circ} / \mathrm{m}$ and $9.74^{\circ} / \mathrm{m}$ $\left(\right.$ mean $\left.=8.88^{\circ} / \mathrm{m}\right)$.

\section{Experiment MoA}

The goal of this experiment was to collect users' RDTs with MoA. Similar to the experiments with MCS, the experiment with MoA also contains three randomly ordered sub-experiments: translation, rotation and curvature.

\subsection{Procedure}

In Experiment MoA, subjects were not constrained in a routine starting position and walking path as imposed by MCS. The settings for this experiment are shown in Table 4. The task for the subjects to accomplish was to adjust gain values until they can no longer feel the discrepancy between the virtual and real paths.

During the test, the subject was given a handheld controller with buttons to adjust the gain in two directions, they can adopt fine tuning with the step value described in Table 4 by clicking the button, or apply quick modifications by pressing and holding the button. There was no imposed starting location for all trials, so the next trial begun immediately after each gain submission. For translation and curvature tests, the subject was instructed to walk back and forth between two target objects. For rotation, a random rotation direction (left or right) was chosen by the program and the subject should follow the given direction.

The angle notation used for the curvature sub-experiment was with respect to an arc length of $3 \mathrm{~m}$, which means the step of gains was $0.33^{\circ}$ direction change per meter, and the curvature gain can vary from -0.29 to 0.29 according to Equation 6.

For the translation and rotation sub-experiments, ascending $\left(g_{\text {init }}<1\right)$ and descending $\left(g_{\text {init }}>1\right)$ trials were presented alternatively, where $g_{\text {init }}$ was the starting gain value. For the curvature sub-experiment, we did not force left-right alternative distribution of the starting gain $g_{\text {init }}$ to prevent learning effect. In all sub-experiments, $g_{\text {init }}$ was a random value chosen to be away from the neutral zone and the lower or upper limits of gains. 
Table 5. The mean and standard deviation for the results of Experiment MoA (only subjects with valid results for MCS were included).

\begin{tabular}{cccc}
\hline & Translation & Rotation & Curvature \\
\hline Number of subjects & 22 & 19 & 19 \\
PSE & $1.01 \pm 0.09$ & $0.98 \pm 0.10$ & $-0.01 \pm 0.03$ \\
IU & $0.22 \pm 0.08$ & $0.26 \pm 0.10$ & $0.15 \pm 0.07$ \\
Upper \& lower bounds & {$[0.90,1.12]$} & {$[0.85,1.11]$} & {$[-0.08,0.06]$} \\
duration (minutes) & $17.0 \pm 5.9$ & $14.4 \pm 6.3$ & $19.8 \pm 8.2$ \\
walking speed (m/s) & $0.54 \pm 0.11$ & $/$ & $0.39 \pm 0.07$ \\
\hline
\end{tabular}

There was no time limit or predefined path for all subjects during the experiment. They can submit the final choice for a trial by pulling the trigger button on the same controller.

\subsection{Results}

All 24 subjects finished successfully all the trials in this experiment, though we put aside the results of the subjects who failed to get valid data with MCS so we can compare the two methods over the same group of subjects. A brief summary of the results for the remaining subjects can be found in Table 5 . The PSE and IU was computed using Equation 4 and 5. The detection threshold $D T_{c}($ left $)=-0.08$ and $D T_{c}$ (right $)=0.06$ correspond respectively to a radius of $12.5 \mathrm{~m}$ and $16.7 \mathrm{~m}$, or $4.58^{\circ} / \mathrm{m}$ and $3.44^{\circ} / \mathrm{m}$.

Since it was difficult to choose a threshold to determine whether a subject was walking, the walking speed shown in Table 5 was the average speed from the start till the end of the experiment for a given subject.

\section{Analyses and Discussions}

In this section, we thoroughly analyzed the data collected in the previous section. All the analyses were performed with $\mathrm{R}^{5}$. The results presented were considered statistically significant when $p \leq 0.05$.

\subsection{Comparison between MCS \& MoA}

We first used Shapiro-Wilk test for normality checking. Several RDT variables from different sub-experiments failed the normality test, therefore we employed Wilcoxon Signed-rank test to compare dependent variables like RDTs, experiment durations and walking speeds of two conditions - MCS and MoA, the results are summarized in Table 6 . We denote $M$ as the median value for a given data set.

Regarding RDTs, the PSE for translation gains obtained with MCS $(M=$ $0.938)$ was smaller than that $(M=1.013)$ of $\operatorname{MoA}(W=65, Z=-1.9966, p<$

\footnotetext{
${ }^{5}$ https://www.r-project.org/
} 

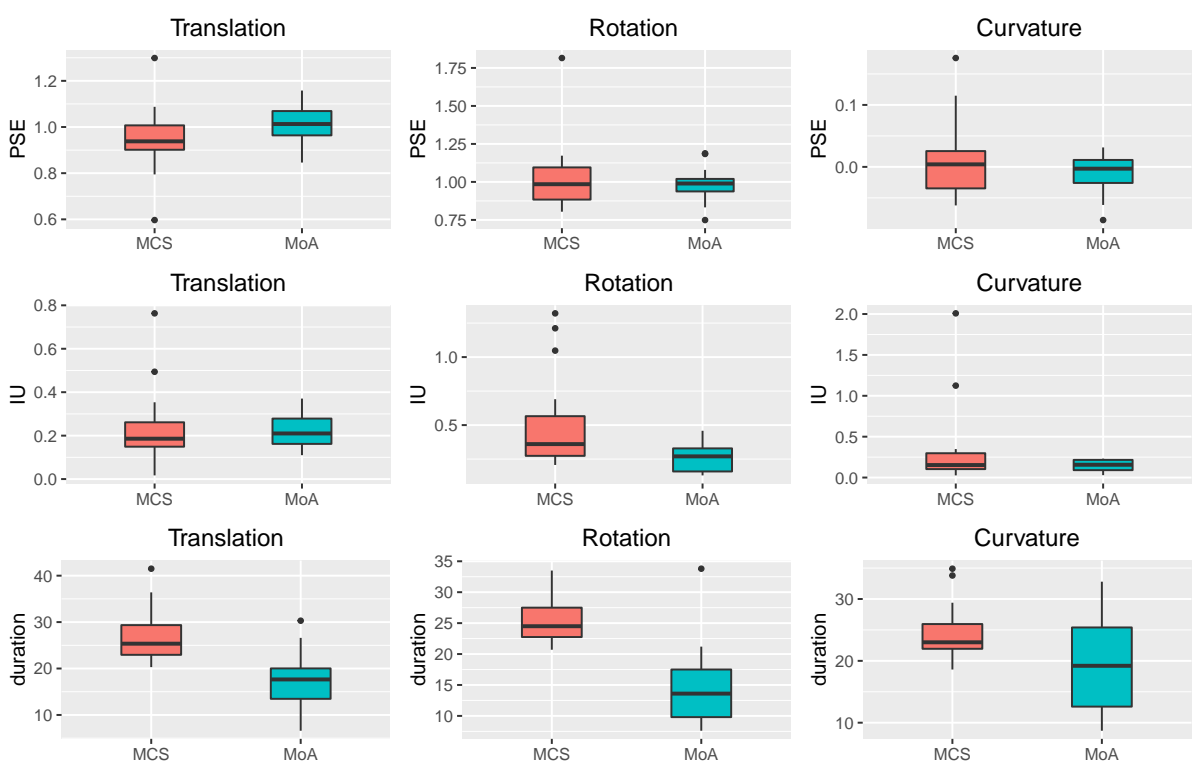

Fig. 4. Comparisons between MCS and MoA on PSE, IU and experiment duration in the translation, rotation and curvature sub-experiments.

Table 6. A summary of comparisons between MCS and MoA ( $\mathrm{n}=$ non significant, * $\left.p \leq 0.05,{ }^{* *} p \leq 0.01\right)$.

\begin{tabular}{cccc}
\hline & Translation & Rotation & Curvature \\
\hline PSE & MCS $<\mathrm{MoA}^{*}$ & $\mathrm{n}$ & $\mathrm{n}$ \\
IU & $\mathrm{n}$ & $\mathrm{MCS}>\mathrm{MoA}^{* *}$ & $\mathrm{n}$ \\
duration & $\mathrm{MCS}>\mathrm{MoA}^{* *}$ & $\mathrm{MCS}>\mathrm{MoA}^{* *}$ & $\mathrm{n}$ \\
avg speed & $\mathrm{MCS}>\mathrm{MoA}^{*}$ & $/$ & $\mathrm{MCS}>\mathrm{MoA}^{* *}$ \\
\hline
\end{tabular}

$0.05, r=0.3)$, but no significant difference was detected between the PSEs of rotation and curvature gains. Moreover, significant difference on IU between $\operatorname{MCS}(M=0.36)$ and $\operatorname{MoA}(M=0.27)$ was found for rotation gains $(W=$ $182, Z=3.5011, p<0.01, r=0.57)$. The boxplots showing the data distributions of PSE and IU for different sub-experiments can be found in Figure 4.

For the experiment duration, we can see from Figure 4 that MoA was indeed more time-efficient than MCS in the translation sub-experiment $\left(M_{M C S}=\right.$ $\left.25.35, M_{M o A}=17.65, W=247, Z=3.9121, p<0.01, r=0.59\right)$, and also in the rotation sub-experiment $\left(M_{M C S}=24.5, M_{M o A}=13.6, W=184, Z=\right.$ $3.5823, p<0.01, r=0.58)$. However, the difference between MCS $(M=23.0)$ and MoA $(M=19.2)$ on experiment duration was not significant in the curvature sub-experiment. Globally, MoA takes on average about 50 minutes to test all three types of gains, which scales down the experiment duration by about $33 \%$ compared to MCS (mean $\approx 75$ minutes). 
Regarding walking speed during experiments, subjects on average took quicker moves with MCS than MoA in the translation $\left(M_{M C S}=0.62, M_{M o A}=0.51, W=\right.$ $185, Z=1.8996, p=0.05, r=0.28)$ and the curvature $\left(M_{M C S}=0.6, M_{M o A}=\right.$ $0.4, W=189, Z=3.7831, p<0.01, r=0.61$ ) sub-experiments (see Figure 5). This difference is possibly due to the uncontrolled walking behavior with MoA, as we observed that some subjects switched constantly between gain adjusting and walking during the sub-experiments with MoA. Overall, subjects walked relatively slow compared to the study of Neth et al. [17] (possibly due to our relatively small physical workspace), which may explain why the detection threshold for curvature gain we got with MCS $\left(v=0.61 \mathrm{~m} / \mathrm{s}, g=8.88^{\circ} / \mathrm{m}\right)$ was higher than what they found $\left(v=0.75 \mathrm{~m} / \mathrm{s}, g=5.42^{\circ} / \mathrm{m}\right)$. However, their finding (i.e. lower walking speed corresponds to higher DT for curvature gain) did not apply to the results of Experiment MoA.
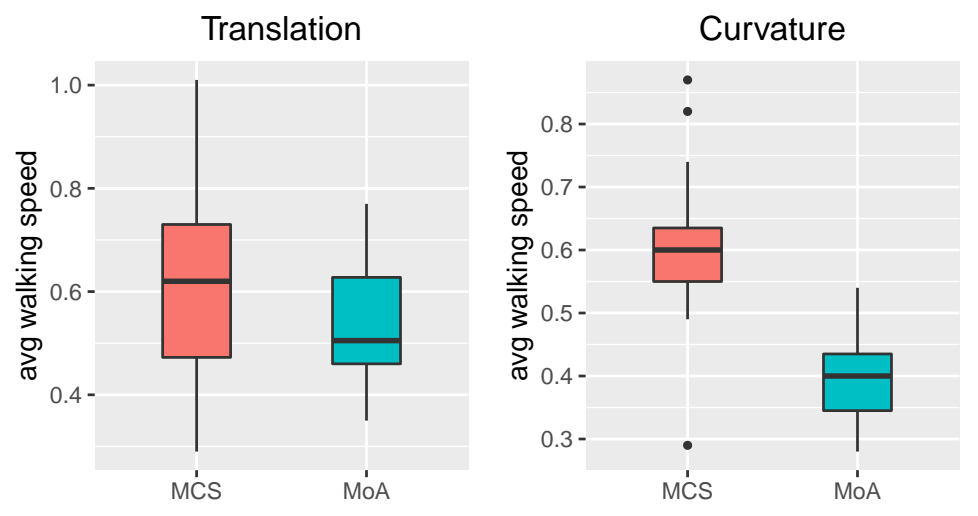

Fig. 5. Comparisons between MCS and MoA on average walking speed in the translation and curvature sub-experiments.

In fact, if we compare the results provided by these two methods at subject level, no apparent rules can be seen because some subjects got very close RDTs in these two experiments while others not. However, when we run statistical analyses on the whole population, significant effects can be found regarding the PSE for translation gains and the IU for rotation gains (see Table 6). The latter might be linked to the initial experiment by Razzaque [23], in which he used MoA for scene rotation detection, but abandoned at last because the pilot subjects found the rotation to be more noticeable while turning the knob (for gain adjustment) in either direction.

It is also interesting to see that the between-subject variances of PSE and IU obtained with MoA were quite small despite the diversity of gain submission strategies among the subjects (some were really quick while others were always in hesitation). Since we were comparing MCS and MoA with the same population, virtual environment and hardware setup, it is safe to claim that the difference 
of results shown in Table 6 came from the sampling methods or the conducted tasks, but no further conclusions can be drawn from the current data.

\subsection{Analyses of MoA}
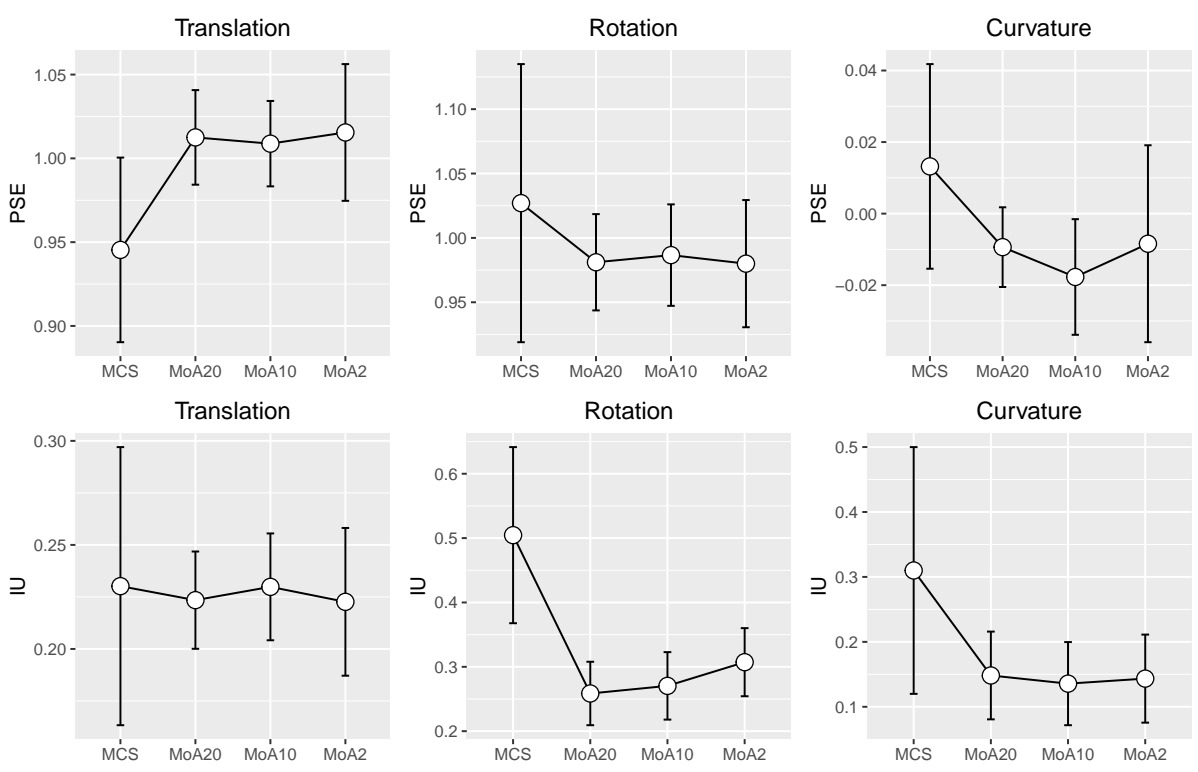

Fig. 6. Further comparisons between MCS and MoA (the number of trials varies from 20 to 2 ) on PSE and IU.

An important question arises when we use MoA is to decide the number of trials. More trials theoretically lead to more robust results as the chance of random choice reduces. However, increasing the number of trials also makes the experiment more time-consuming and burdensome for the participants, which deviates from our initial purpose of using MoA. Here we picked the first 2 and 10 trials per subject respectively, and compared their results with the full-trials (i.e. 20 trials) MoA and MCS. We denote MoA20 as the previously mentioned MoA to facilitate the illustration of its comparison with MoA10 and MoA2.

Since the RDTs did not follow a normal distribution, we applied a withinsubject repeated-measures non-parametric test - Friedman test to assess the influence of testing method (MCS, MoA20, MoA10, MoA2) on RDTs. For posthoc comparisons, we did pairwise comparison with Wilcoxon rank sum tests with Bonferroni correction.

For the translation sub-experiment, no significant effect of testing method on PSE $\left(\chi^{2}(3)=1.78, p=0.62\right)$ or IU $\left(\chi^{2}(3)=2.02, p=0.57\right)$ was found. The significant difference between MCS and MoA20 found in section subsection 6.1 
disappeared with the application of Bonferroni correction, so conclusions on the influence of testing method on PSE should be carefully reconsidered.

For the rotation sub-experiment, there was no effect of testing method on PSE $\left(\chi^{2}(3)=0.79, p=0.85\right)$, but significant effect on IU $\left(\chi^{2}(3)=21.88, p<0.01\right)$. A post-hoc test showed the significant differences between MCS and MoA20 $(p<0.01, r=0.57)$, between MCS and MoA10 $(p<0.01, r=0.51)$ and between MCS and MoA2 $(p<0.01, r=0.41)$.

Regarding the curvature sub-experiment, no significant effect of testing method on $\operatorname{PSE}\left(\chi^{2}(3)=3.76, p=0.29\right)$ and $\mathrm{IU}\left(\chi^{2}(3)=7.61, p=0.055\right)$ was found. We still conducted a post-hoc test for testing method on IU since the $p$-value was close to 0.05 , but no significant effects were found.

The above results show that the number of trials for MoA did not seem to drastically influence the obtained results, except that the between-subject variances (especially for PSE) tend to become smaller as the number of trials increases (see Figure 6).

It is interesting to see that the significant difference between MCS and MoA on IU for rotation gains (Table 6) still holds even when MoA only contains two tests (one ascending trial paired with one descending trial). This means more time can be saved with MoA by reducing the number of trials.

The large variance of experiment duration in the curvature sub-experiment was actually not only due to the diversity of gain submission strategy, but also the difficulties for some subjects to detect the curvature gain. This is possibly because the participants tend to walk in a triangle rather than on an arc when the walking route is short [28].

\subsection{Perception Trait Correlation Test}

Our hypothesis is that users' IUs for redirection are positively related to the scores of tests in group A (in Table 1) since larger non-vision estimation errors should lead to higher tolerance for visual and non-visual sensory conflicts. We also assume that IUs for redirection are positively associated with scores of tests in group B because users with higher visual dependence should be easier to be redirected by visual manipulations.

We used Pearson's product-moment correlation test to examine the correlation between subjects' perception traits and their RDTs given by MCS and MoA. We also created two additional variables - diffPSE and diffIU - to check the potential relationship between the difference of RDTs (given by MCS and MoA) and other existing variables.

The correlation tests showed that subjects' perception traits were neither correlated with their RDTs nor the difference of RDTs in all sub-experiments, which means our hypothesis of a simple linear correlation between the two groups of perceptual abilities and RDTs does not hold. 


\section{Conclusion}

Based on the reported experiments and associated analyses, our primary conclusion is that MoA can provide similar RDT estimates over the same population with MCS except for slightly higher PSE for translation gains and smaller IU for rotation gains. The main advantage of MoA over MCS is that it is highly time-efficient, which can save about $33 \%$ and even more experiment time as the results of MoA remain relatively stable when the number of trials decreases. So $\mathrm{MoA}$ is a good option if we want to quickly assess averaged RDTs for a given population, which can serve as a useful tool for pre-walking calibration or to evaluate the impact of different factors on RDTs in an affordable way.

However, it is still unclear how to explain the difference between results from MoA and MCS at individual level, and how they are correlated with a user's perception trait. The interaction between RDTs and users' intrinsic characteristics such as perception trait should be tested on a bigger yet more heterogeneous population to attempt a personalized model for redirection detection.

Although we had put aside the theoretical difference between MCS and MoA in this paper, we should keep in mind that the underlying psychophysical evaluation method do have an impact on the collected RDTs. Moreover, the RDTs are intrinsically more complex to be explained than other problems studied in the literature of Psychophysics since these usually focus on stimuli getting from the same sensory channel (e.g. the perception of sound, pain, etc.). So inputs from the multi-sensory integration community may help to shed light on this question.

In the future, experiments can be designed using MoA and adaptive methods to clarify the influence of more extrinsic factors (e.g. cognitive load, configuration of the virtual world, real-world ambient noise, etc.) on RDTs. The comparison between MoA and popular adaptive methods can be conducted in a separate study (as there are many variants and parameters for adaptive methods) in order to provide more useful information about the impacts of methodology on RDTs.

\section{References}

1. Azmandian, M.: Design and Evaluation of Adaptive Redirected Walking Systems. Ph.D. thesis (2018)

2. Bourdot, P., Touraine, D.: Polyvalent display framework to control virtual navigations by 6 dof tracking. In: Virtual Reality, 2002. Proceedings. IEEE. pp. 277-278. IEEE (2002)

3. Bruder, G., Interrante, V., Phillips, L., Steinicke, F.: Redirecting walking and driving for natural navigation in immersive virtual environments. IEEE transactions on visualization and computer graphics 18(4), 538-545 (2012)

4. Bruder, G., Lubas, P., Steinicke, F.: Cognitive resource demands of redirected walking. IEEE transactions on visualization and computer graphics 21(4), 539$544(2015)$ 
5. Chen, W., Hu, Y., Ladeveze, N., Bourdot, P.: Quick estimation of detection thresholds for redirected walking with method of adjustment. In: 2019 IEEE Conference on Virtual Reality and 3D User Interfaces (VR). IEEE (2019)

6. Colman, A.M.: A dictionary of psychology. Oxford University Press, USA (2015)

7. Fechner, G.T., Boring, E.G., Howes, D.H., Adler, H.E.: Elements of Psychophysics. Translated by Helmut E. Adler. Edited by Davis H. Howes And Edwin G. Boring, With an Introd. by Edwin G. Boring. Holt, Rinehart and Winston (1966)

8. Gescheider, G.A.: Psychophysics: the fundamentals. Psychology Press (2013)

9. Grechkin, T., Thomas, J., Azmandian, M., Bolas, M., Suma, E.: Revisiting detection thresholds for redirected walking: Combining translation and curvature gains. In: Proceedings of the ACM Symposium on Applied Perception. pp. 113-120. SAP '16, ACM, New York, NY, USA (2016)

10. Guilford, J.P.: Psychometric methods (1954)

11. Hodgson, E., Bachmann, E.: Comparing four approaches to generalized redirected walking: Simulation and live user data. IEEE transactions on visualization and computer graphics 19(4), 634-643 (2013)

12. Kruse, L., Langbehn, E., Stelnlcke, F.: I can see on my feet while walking: Sensitivity to translation gains with visible feet. In: 2018 IEEE Conference on Virtual Reality and 3D User Interfaces (VR). pp. 305-312. IEEE (2018)

13. Kuroda, T., Hasuo, E.: The very first step to start psychophysical experiments. Acoustical Science and Technology 35(1), 1-9 (2014)

14. Langbehn, E., Steinicke, F.: Redirected walking in virtual reality (2018)

15. Matsushima, E.H., da Silva, F.F., de Gouveia, A.P., Pinheiro, R.R., Ribeiro-Filho, N.P., da Silva, J.A.: The psychophysics of visually directed walking. Proceedings of Fechner Day 23(1) (2007)

16. Nescher, T., Huang, Y.Y., Kunz, A.: Planning redirection techniques for optimal free walking experience using model predictive control. In: 3D User Interfaces (3DUI), 2014 IEEE Symposium on. pp. 111-118. IEEE (2014)

17. Neth, C.T., Souman, J.L., Engel, D., Kloos, U., Bulthoff, H.H., Mohler, B.J.: Velocity-dependent dynamic curvature gain for redirected walking. IEEE transactions on visualization and computer graphics 18(7), 1041-1052 (2012)

18. Ngoc, N.T.A., Rothacher, Y., Brugger, P., Lenggenhager, B., Kunz, A.: Estimation of individual redirected walking thresholds using standard perception tests. In: Proceedings of the 22nd ACM Conference on Virtual Reality Software and Technology. pp. 329-330. ACM (2016)

19. Nguyen, A., Rothacher, Y., Lenggenhager, B., Brugger, P., Kunz, A.: Individual differences and impact of gender on curvature redirection thresholds. In: Proceedings of the 15th ACM Symposium on Applied Perception. p. 5. ACM (2018)

20. Nilsson, N., Peck, T., Bruder, G., Hodgson, E., Serafin, S., Suma, E., Whitton, M., Steinicke, F.: 15 years of research on redirected walking in immersive virtual environments. IEEE Computer Graphics and Applications (2018)

21. Paludan, A., Elbaek, J., Mortensen, M., Zobbe, M., Nilsson, N.C., Nordahl, R., Reng, L., Serafin, S.: Disguising rotational gain for redirected walking in virtual reality: Effect of visual density. In: 2016 IEEE Virtual Reality (VR). pp. 259-260 (March 2016)

22. Podlesek, A.i., Komidar, L.: Comparison of three psychophysical methods for measuring displacement in frontal plane motion. Review of psychology 13(1), 51-60 (2006)

23. Razzaque, S.: Redirected Walking. Ph.D. thesis, Chapel Hill, NC, USA (2005)

24. Razzaque, S., Kohn, Z., Whitton, M.C.: Redirected walking. In: Proceedings of Eurographics. vol. 9, pp. 105-106. Citeseer (2001) 
25. Romberg, M.H.: A manual of the nervous diseases of man, vol.2. Sydenham Society (1853)

26. Rothacher, Y., Nguyen, A., Lenggenhager, B., Kunz, A., Brugger, P.: Visual capture of gait in redirected walking. Measuring Behavior 2010 pp. 427-428 (2018)

27. Schmitz, P., Hildebrandt, J., Valdez, A.C., Kobbelt, L., Ziefle, M.: You spin my head right round: Threshold of limited immersion for rotation gains in redirected walking. IEEE transactions on visualization and computer graphics 24(4), 1623$1632(2018)$

28. Steinicke, F., Bruder, G., Jerald, J., Frenz, H., Lappe, M.: Estimation of detection thresholds for redirected walking techniques. IEEE transactions on visualization and computer graphics 16(1), 17-27 (2010)

29. Steinicke, F., Bruder, G., Kohli, L., Jerald, J., Hinrichs, K.: Taxonomy and implementation of redirection techniques for ubiquitous passive haptic feedback. In: Cyberworlds, 2008 International Conference on. pp. 217-223. IEEE (2008)

30. Suma, E.A., Bruder, G., Steinicke, F., Krum, D.M., Bolas, M.: A taxonomy for deploying redirection techniques in immersive virtual environments. In: Virtual Reality Short Papers and Posters (VRW), 2012 IEEE. pp. 43-46. IEEE (2012)

31. Templeman, J.N., Denbrook, P.S., Sibert, L.E.: Virtual locomotion: Walking in place through virtual environments. Presence 8(6), 598-617 (1999)

32. Usoh, M., Arthur, K., Whitton, M.C., Bastos, R., Steed, A., Slater, M., Brooks Jr, F.P.: Walkingi, walking-in-place $\_$flying, in virtual environments. In: Proceedings of the 26th annual conference on Computer graphics and interactive techniques. pp. 359-364. ACM Press/Addison-Wesley Publishing Co. (1999)

33. Walker, J.: Redirected walking in virtual environments. Michigan Technological University (2013)

34. Wier, C.C., Jesteadt, W., Green, D.M.: A comparison of method-of-adjustment and forced-choice procedures in frequency discrimination. Perception \& Psychophysics 19(1), 75-79 (Jan 1976)

35. Witkin, H.A., Asch, S.E.: Studies in space orientation. iv. further experiments on perception of the upright with displaced visual fields. Journal of Experimental Psychology 38(6), 762-782 (1948) 